\title{
Productivity Model for Shale Gas Reservoir with Comprehensive Consid- eration of Multi-mechanisms
}

\author{
Ya Deng ${ }^{1}$, Rui Guo ${ }^{1}$, Zhongyuan $\operatorname{Tian}^{1}$, Cong Xiao ${ }^{2, *}$, Haiying $\operatorname{Han}^{1}$ and Wenhao Tan $^{2}$ \\ ${ }^{1}$ PetroChina Research Institute of Petroleum Exploration \& Development, Beijing 100083, China; ${ }^{2}$ College of Petro- \\ leum Engineering, China University of Petroleum, Beijing 102249, China
}

\begin{abstract}
Multi-stage fracturing horizontal well currently has been proved to be the most effective method to produce shale gas. This method can activate the natural fractures system defined as stimulated reservoir volume (SRV), the remaining region similarly is defined as un-stimulated reservoir volume (USRV). At present, no type curves have been developed for hydraulic fractured shale gas reservoirs in which the SRV zone has triple-porosity dual-depletion flow behavior and the USRV zone has double porosity flow behavior. In this paper, the SRV zone and USRV zone respectively are simplified as cubic triple-porosity and slab dual porosity media. We have established a new productivity model for multifractured horizontal well shale gas with Comprehensive consideration of desorption, diffusion, viscous flow, stress sensitivity and dual-depletion mechanism in matrix. The rate transient responses are inverted into real time space with stehfest numerical inversion algorithm. Type curves are plotted, and different flow regimes in shale gas reservoirs are identified. Effects of relevant parameters are analyzed as well. The whole flow period can be divided into 8 regimes: bilinear flow in SRV; pseudo elliptic flow; dual inter-porosity flow; transitional flow; linear flow in USRV; inter-porosity flow and boundary-dominated flow. The stress sensitivity basically has negative influence on the whole productivity period .The less the value of Langmuir volume and the lager the value of Langmuir pressure, the more lately the inter-porosity flow and boundary-dominated flow occurs. It in concluded that the USRV zone has positive influence on production and could not be ignored.
\end{abstract}

Keywords: Desorption, diffusion, dual inter-porosity, fractured horizontal well, productivity model, shale gas, SRV, stress sensitivity, type curve.

\section{INTRODUCTION}

Shale gas are typical unconventional reservoir due to its ultra-low permeability and porosity. Generally speaking, there is no natural productive capacity for those kinds of reservoirs. Multi-stage fracturing horizontal well currently has been proved to be the most effective way to produce shale gas, and this method can not only create several highconductivity hydraulic fractures, but also activate and connect the existing natural fractures so as to form large spacious network system [1]. The zone containing the main high-conductivity hydraulic fractures and large spacious network system both of which can effectively improve the wells performance is defined as SRV (stimulated reservoir volume), and the remaining zone which hardly influenced by the treatment of hydraulic fracturing is similarly defined as USRV (un-stimulated reservoir volume) [2-4].

At present, a number of scholars have done large amount of researches about transient rate analysis for shale gas, some analytical and semi-analytical solutions are developed as well. Shale gas reservoir is the classical naturally fractured reservoir (NFR) which contains complex natural fractures and ultra-low permeability. In terms of this kinds of reservoirs, Barenblatt (1960), Warren and Root (1963) originally

*Address correspondence to this author at the College of Petroleum Engineering, China University of Petroleum, Beijing 102249, China;

Tel: 18810907235; E-mail: 987558984@qq.com proposed the dual-porosity model which assumed pseudo steady state fluid transfer between matrix and fractures [5, 6], and then Kazemi (1969), de Swaan (1976) and Ozkan et al. (1987) developed some other dual-porosity models for shale gas reservoirs to enrich the former productivity model, these models assumed unsteady-state (transient) flow condition between matrix and fractures [7-9]. However, all of these dual-porosity models neglected the diffusion and adsorption phenomenon in shale gas reservoirs.

Some scholars investigated amount of field production data and found that these dual-porosity models may not be true in actual reservoirs. An improvement to overcome this drawback is to considerate two different fracture systems with different properties. This system is so-called triple porosity system. Al-Ghamdi and Ershaghi (1996) initiatively proposed the dual fracture triple-porosity model for radial flow [10], and then Liu et al. (2003), Wu et al. (2004) and Dreier (2004) enriched the triple-porosity model [11-13], but unfortunately these model still did not considerate the impact of adsorption and diffusion. However, the linear flow stage are apparently identified in some real productivity curves, especially for these fractured reservoirs, therefore, the linear flow models for shale gas are proposed by some scholars. ElBanbi (1998) proposed a linear dual- porosity model in linear fractured reservoirs [14], and originally derived the solutions in Laplace space, but the impact of desorption, diffusion and USRV zone on the production is ignored; Hasan and Al-Ahmadi (2011) proposed a triple-porosity linear flow 
model with consideration of the impact of shale gas desorption and diffusion [15], however, the impact of USRV zone was neglected; Xu et al. (2012) analyzed the effect of USRV zone on shale gas production, at the same time, the impact of desorption diffusion is considered as well [16]; Zhao et al. (2013) proposed triple-porosity spherical flow model for the fractured infinite shale gas reservoirs which considered the impact of diffusion and desorption [17], however, they considered artificial fractures as infinite conductivity.

In terms of these naturally fractured reservoirs, the phenomenon of stress sensitivity is readily observed. Samaniego VF et al. (1980), Raghavan et al. (2004) employed simulation and experiment methods to make some research about the impact of stress sensitivity on the conventional reservoirs $[18,19]$. Pedrosa et al. (1986) first applied the mathematical method to study the stress sensitivity for homogeneous and dual-porosity reservoirs [20]; Wang (2013) develop a dual porosity spherical flowing model with consideration of the stress sensitivity in micro-fractures for shale gas reservoirs [21].

Ezulike Daniel Obinna and Dehghanpour Hassan (2014) first proposed the triple-porosity dual inter-porosity linear flow model [22], that is to say, the gas simultaneously depletes from matrix into micro-fracture and macro-fracture, however, the desorption, diffusion and stress sensitivity in fracture are ignored.

In view of this, shale gas transfer in the reservoir is the result of mutual effects of various percolation mechanisms, and therefore, it is necessary to comprehensively consider the impact of various mechanisms in order to obtain important dynamic parameters for shale gas reservoirs. This paper simplifies the SRV zone and USRV zone as triple-porosity cubic model and dual-porosity slab model respectively, comprehensively taking various mechanisms into account, such as adsorption and diffusion in shale matrix, viscous flow and stress sensitivity in fractures, besides, we assume the gas simultaneously depletes from the matrix into microfractures and macro-fractures in SRV zone and this is the essence of this paper. Laplace transformation, perturbation method are employed to solve this new model. The transient rate responses are inverted into real time space with stehfest numerical inversion algorithm [23]. Type curves are plotted, and different flow regimes in shale gas reservoirs are identified. The effects of relevant parameters are analyzed as well. Besides, this model also compares with numerical simulation and exhibits good agreements.

\section{PRODUCTIVITY MODEL}

\subsection{Physical Model}

The schematic illustration in Fig. (1a) shows a multistage fracturing horizontal well. Multi-stage fracturing shale gas reservoir is divided into SRV zone and USRV zone, SRV zone and USRV zone is simplified as cubic tripleporosity model and dual-porosity slab model. A horizontal well located in the center of a rectangular closed formation producing at constant wellbore pressure. The other assumptions are as follows:

(1) The initial pressure distribution in the reservoir is uniform which equals to $P_{\mathrm{i}}$, the SRV zone contains micro- fractures, macro-fractures and matrix, the USRV zone contains micro-fractures and matrix, the fractures in different zone have different properties.

(2) The macro-fracture is perpendicular to the horizontal well and evenly distributed along the wellbore, the micro-fractures are perpendicular to the macro-fractures as well, the length of reservoir and horizontal wellbore are equivalent, the length of micro-fracture and the width of reservoir respectively equals to $y_{\mathrm{f}}$ and $y_{\mathrm{e}}$.

(3) Macro-fractures have finite conductivity and are assumed to be penetrated fully, considering stress sensitivity in macro-fractures.

(4) Flowing is sequential from one medium to another medium. In the SRV zone, only fluid flow from macrofractures to wellbore is considered; The shale gas simultaneously deplete from matrix into micro-fractures and macro-fractures with pseudo-steady state inter-porosity flow; the fluid flow between micro-fractures and macrofractures is unsteady state flowing. In the USRV zone, the fluid flowing from matrix to the fractures is pseudosteady state inter-porosity flow; the connection between SRV zone and USRV zone via the macro-fractures in SRV and micro-fractures in USRV.

(5) Slightly compressible shale gas and compressibility coefficient is constant;

(6) Shale gas desorption and diffusion respectively meets the Langmuir isotherm equation and the first law of diffusion;

(7) The impact of gravity and capillary pressure is ignored.

This paper considers the simultaneous depletion from matrix into micro-fractures and macro-fractures. To analyze this flow process conveniently, the matrix in SRV zone is artificially divided into two distinct segments which have different permeability and porosity ratio respectively. The schematic illustration in Fig. (1b) shows the depletion process from matrix to micro-fractures and macro-fractures in SRV zone.

\subsection{Mathematical Model}

Based on the mass balance principle, the governing equations respectively in micro-fractures, matrix, and macrofractures in SRV zone and USRV zone with consideration of adsorption, diffusion, viscous flow and stress sensitivity are as follows:

SRV Zone:

Macro-fracture:

$$
\begin{gathered}
\frac{\partial^{2} \phi_{F}}{\partial y^{2}}+\beta\left(\frac{\partial \phi_{F}}{\partial y}\right)^{2}-\left.\frac{1}{L_{F} / 2} \frac{k_{f 1}}{k_{F i}} e^{\beta\left(\phi_{i}-\phi_{F}\right)} \frac{\partial \phi_{f 1}}{\partial x}\right|_{x=L_{F} / 2}+ \\
2 \alpha_{1} e^{\beta\left(\phi_{i}-\phi_{F}\right)} \frac{k_{m 1}}{k_{F i}}\left(\phi_{m 1}-\phi_{F}\right)=e^{\beta\left(\phi_{i}-\phi_{F}\right)} \frac{\left(\varphi \mu c_{t}\right)_{F}}{k_{F i}} \frac{\partial \phi_{F}}{3.6 \partial t}
\end{gathered}
$$

Micro-fracture:

$$
\frac{\partial^{2} \phi_{f 1}}{\partial x^{2}}+2 \alpha_{2} \frac{k_{m 2}}{k_{f 1}}\left(\phi_{m 2}-\phi_{f 1}\right)=\frac{\left(\varphi \mu c_{t}\right)_{f 1}}{k_{f 1}} \frac{\partial \phi_{f 1}}{3.6 \partial t}
$$




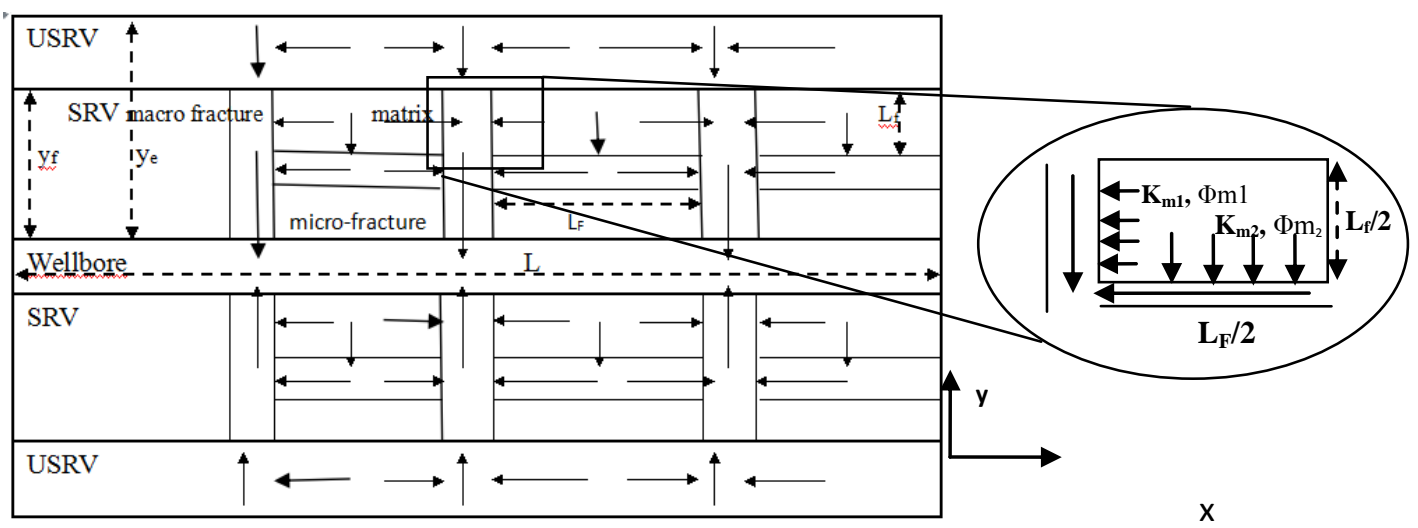

(a)

(b)

Fig. (1). (a) The illustration of multi-fractured horizontal well (b) The two segments of matrix in SRV zone.

The matrix in SRV zone are divided into two segments, the segment denoted as matrix-1 which permeability and porosity equal to $k_{\mathrm{m} 1}$ and $\Phi m_{1}$ depletes into macro-fractures and another segment denoted as matrix-2 which permeability and porosity equal to $k_{\mathrm{m} 2}$ and $\Phi m_{2}$ depletes into microfractures. Therefore, the governing equations of these two segments are as follows:

Matrix-1:

$-2 \alpha_{1} \frac{k_{m 1}}{k_{F i}}\left(\phi_{m 1}-\phi_{F}\right)=\frac{\left(\varphi \mu c_{t}\right)_{m 1}}{k_{F i}} \frac{\partial \phi_{m 1}}{3.6 \partial t}+$

$\frac{\mu Z p_{s c} T V_{L} P_{L}}{T_{s c} k_{F i} \bar{p}\left(p_{L}+\bar{p}\right)^{2}} \frac{\partial \phi_{m 1}}{3.6 \partial t}$

Matrix-2:

$-2 \alpha_{2} \frac{k_{m 2}}{k_{f 1}}\left(\phi_{m 2}-\phi_{f 1}\right)=\frac{\left(\varphi \mu c_{t}\right)_{m 2}}{k_{f 1}} \frac{\partial \phi_{m 2}}{3.6 \partial t}+$

$\frac{\mu Z p_{s c} T V_{L} P_{L}}{T_{s c} k_{f 1} \bar{p}\left(p_{L}+\bar{p}\right)^{2}} \frac{\partial \phi_{m 2}}{3.6 \partial t}$

USRV Zone:

Fracture:

$$
\frac{\partial^{2} \phi_{f 2}}{\partial y^{2}}+2 \alpha_{3} \frac{k_{m 3}}{k_{f 2}}\left(\phi_{m 3}-\phi_{f 2}\right)=\frac{\left(\varphi \mu c_{t}\right)_{f 2}}{k_{f 2}} \frac{\partial \phi_{f 2}}{3.6 \partial t}
$$

Matrix:

$-2 \alpha_{3} \frac{k_{m 3}}{k_{f 2}}\left(\phi_{m 3}-\phi_{f 2}\right)=\frac{\left(\varphi \mu c_{t}\right)_{m 3}}{k_{f 2}} \frac{\partial \phi_{m 3}}{3.6 \partial t}+$

$\frac{\mu Z p_{s c} T V_{L} P_{L}}{T_{s c} k_{f 1} \bar{p}\left(p_{L}+\bar{p}\right)^{2}} \frac{\partial \phi_{m 3}}{3.6 \partial t}$

initial condition:

$$
\begin{aligned}
& \phi_{F}(y, 0)=\phi_{i} \\
& \phi_{f 1}(x, 0)=\phi_{f 2}(y, 0)=\phi_{i} \\
& \phi_{m 1}(x, 0)=\phi_{m 2}(y, 0)=\phi_{m 3}(y, 0)=\phi_{i}
\end{aligned}
$$

Inner boundary condition:

$\varphi_{F}(0, t)=\varphi_{w f}$

Interface condition:

$\varphi_{F}\left(y_{f}, t\right)=\varphi_{f 2}\left(y_{f}, t\right)$

$\left.k_{f 2} \frac{\partial \varphi_{f 2}}{\partial y}\right|_{y=y_{f}}=\left.e^{-\beta\left(\varphi_{i}-\varphi_{F}\right)} k_{F i} \frac{\partial \varphi_{F}}{\partial y}\right|_{y=y_{f}}$

Inner zone micro-fracture:

$\left.\frac{\partial \varphi_{f 1}}{\partial x}\right|_{x=0}=0, \varphi_{f 1}\left(L_{F} / 2, t\right)=\varphi_{F}$

Outer boundary condition:

$\left.\frac{\partial \phi_{f 2}}{\partial y}\right|_{y=y_{e}}=0$

To simplify these equations, some dimensionless variables are defined (seen from appendix A) and substituting these dimensionless variables into equation 4-15, the dimensionless governing equations are as follows:

SRV Zone:

Macro-fracture:

$\frac{\partial^{2} \phi_{D F}}{\partial y_{D}^{2}}-\beta_{D}\left(\frac{\partial \phi_{D F}}{\partial y_{D}}\right)^{2}-\left.\frac{\lambda_{f 1-F}}{3} e^{\beta_{D} \phi_{D F}} \frac{\partial \phi_{D f 1}}{\partial x_{D}}\right|_{x_{D}=1}+$
$2 \lambda_{m 1-F} e^{\beta_{D} \phi_{D F}}\left(\phi_{D m 1}-\phi_{D F}\right)=e^{\beta_{D} \phi_{D F}} \omega_{F} \frac{\partial \phi_{D F}}{\partial t_{D}}$

Micro-fracture:

$\frac{\partial^{2} \phi_{D f 1}}{\partial x_{D}^{2}}+2 \lambda_{m 2-f 1} \eta\left(\phi_{D m 2}-\phi_{D f 1}\right)=\omega_{f 1} \eta \frac{\partial \phi_{D f 1}}{\partial t_{D}}$

Matrix-1:

$-2 \lambda_{m 1-F}\left(\phi_{D m 1}-\phi_{D F}\right)=\omega_{m 1} \frac{\partial \phi_{D m 1}}{\partial t_{D}}+\omega_{d} \frac{\partial \phi_{D m 1}}{\partial t_{d}}$ 
Matrix-2:

$-2 \lambda_{m 2-f 1}\left(\phi_{D m 2}-\phi_{D f 1}\right)=\omega_{m 2} \frac{\partial \phi_{D m 2}}{\partial t_{D}}+\omega_{d} \frac{\partial \phi_{D m 2}}{\partial t_{D}}$

USRV Zone:

Fracture:

$\frac{\partial^{2} \phi_{D f 2}}{\partial y_{D}^{2}}+2 \lambda_{m 3-f 2}\left(\phi_{D m 3}-\phi_{D f 2}\right) \tau=\omega_{f 2} \sigma \tau \frac{\partial \phi_{f 2}}{\partial t_{D}}$

Matrix:

$-2 \lambda_{m 3-f 2}\left(\phi_{D m 3}-\phi_{D f 2}\right)=\omega_{m 3} \sigma \frac{\partial \phi_{D m 3}}{\partial t_{D}}+\omega_{d} \frac{\partial \phi_{D m 3}}{\partial t_{D}}$

Inner boundary condition:

$\phi_{D F}\left(0, t_{D}\right)=1$

Interface condition:

$\phi_{D F}\left(y_{D f}, t_{D}\right)=\phi_{D f 2}\left(y_{D f}, t_{D}\right)$

$\left.k_{f 2} \frac{\partial \phi_{D f 2}}{\partial y_{D}}\right|_{y_{D}=y_{f} / \sqrt{A_{c w}}}=\left.k_{F i} e^{-\beta_{D} \phi_{D F}} \frac{\partial \phi_{D F}}{\partial y_{D}}\right|_{y_{D}=y_{f} / \sqrt{A_{c w}}}$

Inner Zone fracture:

$\left.\frac{\partial \phi_{D f 1}}{\partial x_{D}}\right|_{x_{D}=0}=0, \phi_{D f 1}\left(x_{D}=1, t_{D}\right)=\phi_{D F}$

Outer boundary condition:

$\left.\frac{\partial \phi_{D f 2}}{\partial y_{D}}\right|_{y_{D}=y_{e}} / \sqrt{A_{c w}}=0$

It's not difficult to find that the macro-fracture equation contains strong nonlinear, the perturbation technology and the Presoda transformation are applied to linearize this equation [20], the formula is as follows:

$\phi_{D F}\left(y_{D}, t_{D}\right)=-\frac{1}{\beta_{D}} \ln \left[1-\beta_{D} \zeta\left(y_{D}, t_{D}\right)\right]$

$\psi_{f D}\left(r_{D}, t_{a D}\right)=-\frac{1}{\zeta_{D}} \ln \left[1-\zeta_{D} \eta\left(r_{D}, t_{a D}\right)\right]$

According to the theory conducted by Wang (2013) [24] and Presoda (1986), performing a parameter perturbation in $\zeta_{D}$ by defining the following series:

$$
\begin{aligned}
& \eta=\eta_{0}+\zeta_{D} \eta_{1}+\zeta_{D}^{2} \eta_{2}+\zeta_{D}^{3} \eta_{3}+\ldots \\
& -\frac{1}{\zeta_{D}} \ln \left[1-\zeta_{D} \eta\left(r_{D}, t_{a D}\right)\right]=\eta\left(r_{D}, t_{a D}\right)+ \\
& \frac{1}{2} \zeta_{D} \eta^{2}\left(r_{D}, t_{a D}\right)+\frac{1}{6} \zeta_{D} \eta^{3}\left(r_{D}, t_{a D}\right)+\ldots
\end{aligned}
$$

$$
\begin{aligned}
& \frac{1}{1-\zeta_{D} \eta\left(r_{D}, t_{a D}\right)}=1+\zeta_{D} \eta\left(r_{D}, t_{a D}\right)+ \\
& \zeta_{D}^{2} \eta\left(r_{D}, t_{a D}\right)+\zeta_{D}^{3} \eta\left(r_{D}, t_{a D}\right)+\ldots
\end{aligned}
$$

Finally, the final governing equation of SRV zone and USRV zone can be changed into the following profiles.

SRV Zone:

Macro-fracture:

$\frac{\partial^{2} \zeta}{\partial y_{D}^{2}}-\left.\frac{\lambda_{f 1-F}}{3} \frac{\partial \phi_{D f 1}}{\partial x_{D}}\right|_{x_{D}=1}+2 \lambda_{m 1-F}\left(\phi_{D m 1}-\phi_{D F}\right)=\omega_{F} \frac{\partial \zeta}{\partial t_{D}}$

Micro-fracture:

$\frac{\partial^{2} \phi_{D f 1}}{\partial x_{D}^{2}}+2 \lambda_{m 2-f 1} \eta\left(\phi_{D m 2}-\phi_{D f 1}\right)=\omega_{f 1} \eta \frac{\partial \phi_{D f 1}}{\partial t_{D}}$

Matrix-1:

$-2 \lambda_{m 1-F}\left(\phi_{D m 1}-\phi_{D F}\right)=\omega_{m 1} \frac{\partial \phi_{D m 1}}{\partial t_{D}}+\omega_{d} \frac{\partial \phi_{D m 1}}{\partial t_{d}}$

Matrix-2:

$-2 \lambda_{m 2-f 1}\left(\phi_{D m 2}-\phi_{D f 1}\right)=\omega_{m 2} \frac{\partial \phi_{D m 2}}{\partial t_{D}}+\omega_{d} \frac{\partial \phi_{D m 2}}{\partial t_{D}}$

USRV Zone:

Fracture:

$\frac{\partial^{2} \phi_{D f 2}}{\partial y_{D}^{2}}+2 \lambda_{m 3-f 2}\left(\phi_{D m 3}-\phi_{D f 2}\right) \tau=\omega_{f 2} \sigma \tau \frac{\partial \phi_{D f 2}}{\partial t_{D}}$

Matrix:

$-2 \lambda_{m 3-f 2}\left(\phi_{D m 3}-\phi_{D f 2}\right)=\omega_{m 3} \sigma \frac{\partial \phi_{D m 3}}{\partial t_{D}}+\omega_{d} \frac{\partial \phi_{D m 3}}{\partial t_{D}}$

Inner boundary condition:

$\zeta=\frac{1-e^{-\beta_{D}}}{\beta_{D}}$

Interface condition:

$\zeta\left(y_{D f}, t_{D}\right)=\phi_{D f 2}\left(y_{D f}, t_{D}\right)$

$\left.k_{f 2} \frac{\partial \phi_{D f 2}}{\partial y_{D}}\right|_{y_{D}=y_{f} / \sqrt{A_{c w}}}=\left.k_{F i} \frac{\partial \zeta}{\partial y_{D}}\right|_{y_{D}=y_{f} / \sqrt{A_{c w}}}$

Inner zone fracture:

$\left.\frac{\partial \phi_{D f 1}}{\partial x_{D}}\right|_{x_{D}=0}=0, \phi_{D f 1}\left(x_{D}=1, t_{D}\right)=\zeta$

Outer boundary condition:

$\left.\frac{\partial \phi_{D f 2}}{\partial y_{D}}\right|_{y_{D}=y_{e} / \sqrt{A_{c w}}}=0$ 


\subsection{Model Solution}

The Laplace transformation is used to solve equation 2535 , the details of this process are listed in appendix B. The final solution of these equations in Laplace space is as follows:

$\overline{q_{D}}=-\frac{b \sinh \left(\sqrt{f_{F}} y_{f} / \sqrt{A_{c w}}\right)-a \cosh \left(\sqrt{f_{F}} y_{f} / \sqrt{A_{c w}}\right)}{a \sinh \left(\sqrt{f_{F}} y_{f} / \sqrt{A_{c w}}\right)-b \cosh \left(\sqrt{f_{F}} y_{f} / \sqrt{A_{c w}}\right)} \frac{1-e^{-\beta_{D}}}{s \beta_{D}} \sqrt{f_{F}}$

$a=\frac{k_{f 2} \sqrt{f_{f 2}}}{k_{F} \sqrt{f_{F}}}\left[\sinh \left(\sqrt{f_{f 2}} y_{f} / \sqrt{A_{c w}}\right)-\cosh \left(\sqrt{f_{f 2}} y_{f} / \sqrt{A_{c w}}\right) \tanh \left(\sqrt{f_{f 2}} y_{e} / \sqrt{A_{c w}}\right)\right]$

Where:

$b=\left[\cosh \left(\sqrt{f_{f 2}} y_{f} / \sqrt{A_{c w}}\right)-\sinh \left(\sqrt{f_{f 2}} y_{f} / \sqrt{A_{c w}}\right) \tanh \left(\sqrt{f_{f 2}} y_{e} / \sqrt{A_{c w}}\right)\right]$

However, to analyze the impact of relative parameters and identify the shale gas flowing regimes, the transient rate responses are inverted into real time space with stehfest numerical inversion algorithm.

\section{NUMERICAL SIMULATION VALIDATION}

Numerical models were used to validate this new analytical solution proposed in the paper. This validation process is operated in the commercial software-CMG. We assume that the rate of water is ignored and gas is the only phase existing in the reservoir. The basic parameters are listed in the Table 1. The radial coordinate is converted into $\mathrm{x}-\mathrm{y}$ coordinate, and the value of the permeability of these two directions is different based on the flowing regimes. In SRV zone, the vertical permeability of some grids which are located near the wellbore is assumed to be very high so as to ensure the facts that the gas can flow into the wellbore. It is difficult to simulated the adsorption used CMG, for simplicity, the adsorbed gas is not included. We focus on the tendency of curves.

For gas case, as seen in Fig. (2), the simulation data from CMG are converted into the formation of dimensionless time and dimensionless rate for the convenience of analysis. It is concluded that the curve is in good agreement with the analytical solution at the stage $\ominus$ which represents the flowing regime in fractures in SRV zone and stage $\circledast$ which represents the flowing regime in fractures in USRV zone, while it deviates a lot at the stage $\ominus$ which represents the flowing regime in matrix in SRV zone and stage (4) which represents the flowing regime in matrix in USRV zone. The main reason for the deviation is that adsorption is neglected in simulation model. The deviation also could be caused by using the inappropriate average pressure to calculate the pseudo pressure. At the very early times, the flow is fracturedominated flowing regime in SRV zone, the adsorptive gas has not desorbed from the surface of matrix, so the predictive results are similar with those from the numerical simulation (as seen in stage $\Theta$ and $\circledast$ ). However, at the middle times and late times, the flow is matrix-dominated and boundary-dominated flowing regime, and the situation is different. The adsorptive gas can desorb from the surface of matrix and depletes into fractures, this process can increase the shale gas production (as seen in stage $\ominus$ and (4)).
Table 1. The value of input parameters (constant production pressure).

\begin{tabular}{|c|c|}
\hline Parameters & Value \\
\hline Initial pressure,$P_{\mathrm{i}}(\mathrm{MPa})$ & 48 \\
\hline Downhole pressure, $P_{\mathrm{wf}}(\mathrm{MPa})$ & 25 \\
\hline Formation temperature,$T(\mathrm{~K})$ & 333 \\
\hline Horizontal length, $L(\mathrm{~m})$ & 2000 \\
\hline Formation thickness, $h(\mathrm{~m})$ & 100 \\
\hline Macro-fracture length, $y_{\mathrm{f}}(\mathrm{m})$ & 200 \\
\hline Outer boundary space, $y_{\mathrm{e}}(\mathrm{m})$ & 1000 \\
\hline Total compressibility of macro-fracture in $\mathrm{SRV}, C \mathrm{t}_{\mathrm{F}}\left(\mathrm{MPa}^{-1}\right)$ & $10^{-3}$ \\
\hline Total compressibility of micro-fracture in $\mathrm{SRV}, C \mathrm{t}_{\mathrm{f} 1}\left(\mathrm{MPa}^{-1}\right)$ & $5 * 10^{-4}$ \\
\hline Total compressibility of matrix, $C \mathrm{tm}\left(\mathrm{MPa}^{-1}\right)$ & $5 * 10^{-4}$ \\
\hline Total compressibility of fracture in USRV, $C \mathrm{t}_{\mathrm{f} 2}\left(\mathrm{MPa}^{-1}\right)$ & $5 * 10^{-4}$ \\
\hline Porosity of macro-fracture in SRV, $\Phi_{\mathrm{F}}$ & 0.001 \\
\hline Porosity of micro-fracture in SRV, $\Phi_{\mathrm{f} 1}$ & 0.005 \\
\hline Porosity of matrix-1 in SRV, $\Phi_{\mathrm{m} 1}$ & 0.05 \\
\hline Porosity of matrix-2 in SRV, $\Phi_{\mathrm{m} 2}$ & 0.05 \\
\hline Porosity of fracture in USRV, $\Phi_{\mathrm{f} 2}$ & 0.005 \\
\hline Porosity of matrix in USRV, $\Phi_{\mathrm{m} 3}$ & 0.05 \\
\hline Initial permeability of macro-fracture in $\mathrm{SRV}, k_{\mathrm{Fi}}(\mathrm{D})$ & $10^{-3}$ \\
\hline Permeability of micro-fracture in SRV, $k_{\mathrm{fl}}(\mathrm{D})$ & $\begin{array}{l}10^{-4} \\
1010\end{array}$ \\
\hline Permeability of matrix-1 in SRV, $k_{\mathrm{m} 1}(\mathrm{D})$ & $10^{-5}$ \\
\hline Permeability of matrix-2 in SRV, $k_{\mathrm{m} 2}(\mathrm{D})$ & $10^{-6}$ \\
\hline Permeability of fracture in USRV,$k_{\mathrm{f} 2}(\mathrm{D})$ & $10^{-5}$ \\
\hline Permeability of matrix in USRV,$k_{\mathrm{m} 3}(\mathrm{D})$ & $10^{-6}$ \\
\hline
\end{tabular}

\section{TYPE CURVES AND DISCUSSIONS}

In this paper, we make some assumptions that SRV zone is cubic triple-porosity model and USRV zone is slab dualporosity model. Some characteristic parameters of these kinds of shale gas reservoirs include: $\omega_{\mathrm{F}}, \omega_{\mathrm{f} 1}, \omega_{\mathrm{m} 1}, \omega_{\mathrm{m} 2}, \omega_{\mathrm{f} 2}$, $\omega_{\mathrm{m} 3}$. However, if these parameters are equal to some special value, the new model proposed in this paper can be changed into some other familiar models. For example, $\omega_{\mathrm{f} 1}$ is equal to 0 , the model of SRV zone can be dual porosity slab model; when the $\omega_{\mathrm{m} 3}$ equals to 0 , USRV zone can be simplified as homogeneous model; $\omega_{\mathrm{m} 1}=0$, the gas only depletes from the matrix to micro-fractures in SRV zone (Hasan A. AlAhmadi, 2011), in another words, the new model can be similar with the triple-linear model proposed by El-Banbi (1988) as well; $\omega_{\mathrm{m} 2}=0$, SRV zone can be simplified as dualporosity model (Xu et al, 2013). In short, this new model has universal application to different formations when these characteristic parameters are equal to different values. It is 


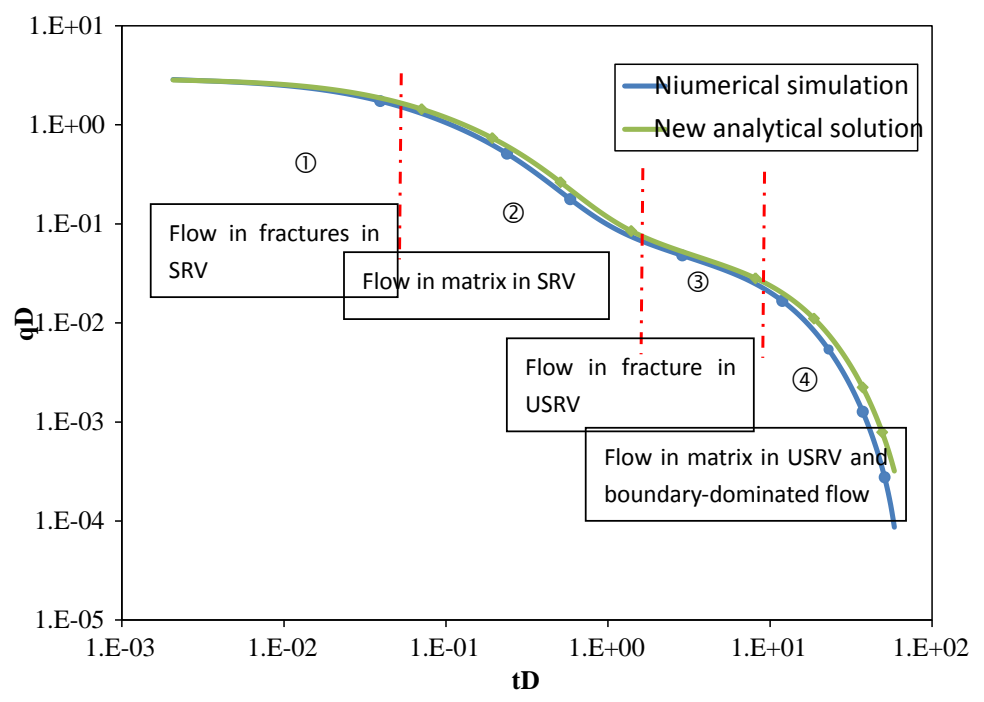

Fig. (2). Comparison of numerical simulation and analytical solution.

worth mentioning that the stress sensitivity in the macrofractures is considered, this consideration extremely matches the real production condition in fractured reservoirs, especially for the ultra-low permeability shale gas reservoirs. The mechanism of the stress sensitivity will be analyzed in the following sections.

Combined with stehfest numerical inversion algorithm, the type curves concerning dimensionless rate derivative $q_{D}{ }^{\prime}$ with respect to $t_{D}$ and dimensionless rate $q_{D}$ with respect to $t_{D}$ are plotted under different condition of formation properties, eight flow regimes can be easily observed by analyzing the following type curves Seen from Fig. (3):

Regime I: bilinear flow regime. The value of the slope of the rate derivative curve is equal to $-1 / 4$. This flow regime occurs between the micro-fracture and macro-fractures in SRV zone, because of the existence of micro-fractures in SRV zone, it is difficult to find the single linear flow in the macro-fractures in the SRV zone, especially, when the permeability of macro-fractures is big.

Regime II: pseudo elliptic flow regime. The value of the slope of the rate derivative curve equals to $-1 / 3$, this is the transitional flow stage from the bilinear flow regime between the micro-fracture and macro-fractures to linear flow in micro-fractures in SRV zone.

Regime III: linear flow regime. The value of the slope of the rate derivative curve is equal to $-1 / 2$. This is the linear flow which occurs in the micro-fractures of SRV zone.

Regime IV: pseudo-steady state inter-porosity regime. Two consecutive incomplete concave dips denoted with a circle can be ambiguously observed, these two sections respectively present two different inter-porosity flow regimes which simultaneously occur from matrix to micro-fractures and macro-fractures in SRV zone;

Regime V: transition flow regime. The pressure wave reaches to the interface between SRV zone and USRV zone, USRV zone supply to SRV zone, the decline ratio of rate decreases as well. As a result, the rate derivative curves elevate and the transition flow regime occurs between these two zones.
Regime VI: linear flow regime. The value of the slope of the rate derivative curve is equal to $-1 / 2$. This regime mainly occurs in the fractures in USRV zone so that the shale gas stored in the USRV zone can deplete from USRV zone to SRV zone via interface.

Regime VII: pseudo-steady state inter-porosity flowing regime. The shape of rate derivative curve likes a "concave" which is typical feature of pseudo-steady state inter-porosity. This regime mainly occurs between matrix and fractures in USRV zone;

Regime VIII: Boundary-dominated flow regime. At this time, the boundary has influence on dynamic production of well, the rate and rate derivative curve decrease rapidly.

The paper considers the impact of stress sensitivity in the macro-fractures in SRV zone. Fig. (4) shows that stress sensitivity has great negative influence on horizontal well performance during the whole productivity period. However, with the extension of production time, the negative impact on rate becomes less and less, especially during the boundary-dominated flow stage and the negative impact basically vanishes. The permeability of macro-fractures in SRV zone consecutively decreases with the reduction of pressure, when the pressure wave has reached to the outer boundary, the permeability of the macro-fractures is so small that the influence of stress sensitivity can be ignored. Besides, with the increasing of stress sensitivity coefficient $\left(\beta_{D}=0, \beta_{D}=0.8\right.$, $\beta_{\mathrm{D}}=1.5$ ), dimensionless rate curve (Fig. 4a) and dimensionless rate derivative curve (Fig. 4b) simultaneously descend. By analyzing the mechanisms, the more the pressure reduces in macro-fractures, the more severely the macro-fractures close, as a result, the permeability of macro-fracture rapidly decreases.

Two most important characteristic parameters are Langmuir pressure and Langmuir volume which reflect the feature of adsorption. $P_{\mathrm{L}}$ and $V_{\mathrm{L}}$ primarily affect the stage of pseudo-steady state inter-porosity between matrix and fracture in SRV zone, linear flow in USRV zone and outer boundary-dominated flow. The larger the value of $P_{\mathrm{L}}\left(P_{\mathrm{L}}\right.$ perspective is equals to $2 \mathrm{MPa}, 5 \mathrm{MPa}$ and $10 \mathrm{MPa}$ ) or the 


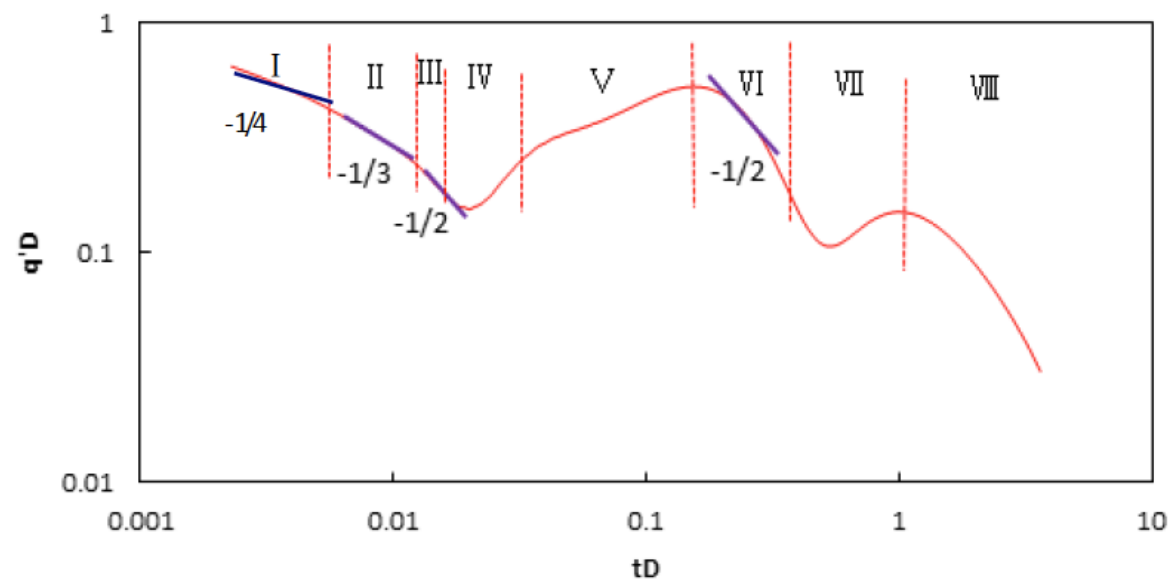

Fig. (3). Type curve and the division of flow regime.

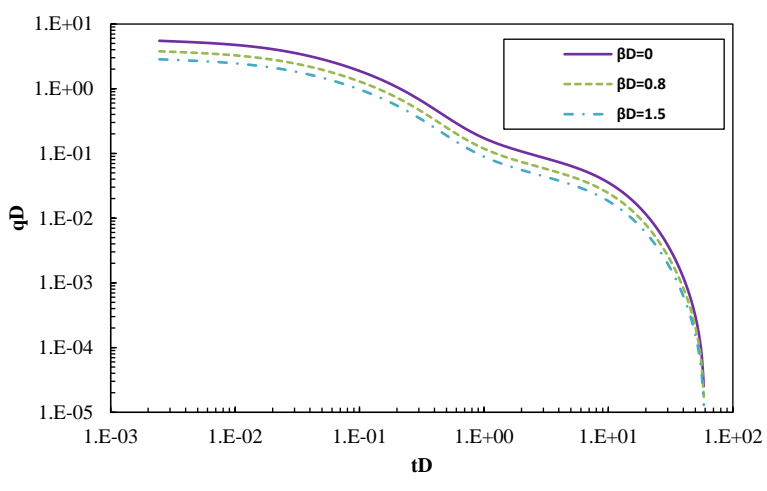

(a)

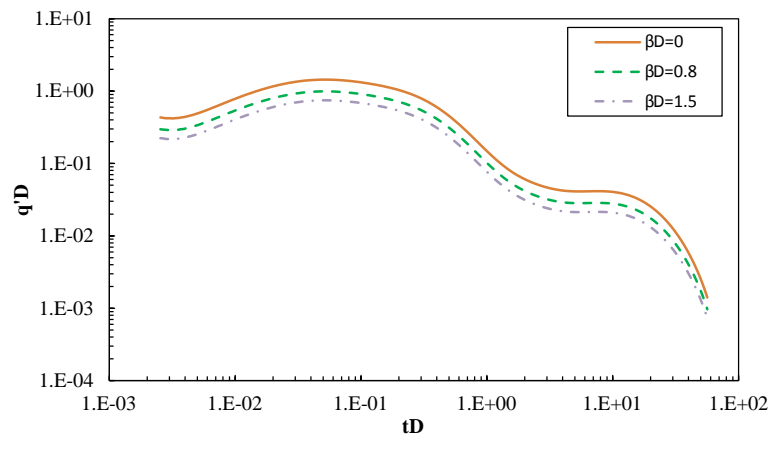

(b)

Fig. (4). Type curve of different stress sensitivity coefficient: (a) dimensionless rate curves; (b) dimensionless rate derivative curves.

smaller the value of $V_{\mathrm{L}}\left(\mathrm{V}_{\mathrm{L}}\right.$ perspective is equal to $5 \mathrm{sm}^{3} / \mathrm{m}^{3}$, $10 \mathrm{sm}^{3} / \mathrm{m}^{3}, 15 \mathrm{sm}^{3} / \mathrm{m}^{3}$ ), the less the shale gas rate during the whole process of productivity period, and the later the stage of pseudo-steady state inter-porosity between matrix and fractures in SRV zone occurs, and linear flow in USRV zone and outer boundary-dominated flow occurs.

From the analysis of micro-mechanisms, the larger the value of Langmuir volume is and the more the content of adsorbed gas are. When the pressure is smaller than Langmuir pressure, the adsorbed gas starts to desorb from the surface of matrix and spread into fractures so that the decline ratio of pressure and rate slows down. At the same time, due to inter-porosity flowing between matrix and fracture, the negative of outer boundary will delate lately, in another word, the dimensionless rate derivative curve wholly moves right (Fig. 5a, d). In short, due to the gas desorption and diffusion, the decline ratio of pressure becomes slow and the rate is less affected by outer boundary. When shale gas desorption reaches a certain level, the amount of desorption is insufficient to cover the impact of outer boundary, dimensionless rate derivative curve moves downward (Fig. 5b, c).

The paper assumes that gas simultaneously depletes from matrix into micro-fractures and macro-fractures in SRV zone. The matrix is divided into two segments, where, the segment denoted as matrix-1 which permeability and porosity equal to $k_{\mathrm{m} 1}$ and $\Phi_{\mathrm{m} 1}$ depletes into macro-fractures and another segment denoted as matrix 2 which permeability and porosity equal to $k_{\mathrm{m} 2}$ and $\Phi_{\mathrm{m} 2}$ depletes into micro-fractures. We introduce the pore volume ratio denoted as $\chi$ which equals to $\Phi_{\mathrm{m} 1} /\left(\Phi_{\mathrm{m} 1}+\Phi_{\mathrm{m} 2}\right)$, and $\chi=0, \chi=1,0<\chi<1$ respectively represents: (1) the matrix only depletes into micro-fractures; (2) the matrix only depletes into macro-fractures; (3) matrix simultaneously depletes from matrix into micro-fractures and macro-fractures. The type curves (Fig. 6) under different value of $\chi, k_{\mathrm{m} 1}$ and $k_{\mathrm{m} 2}$ are plotted. Some conclusions can be obtained via the analysis of curves: (1) $k_{\mathrm{m} 1}>\mathrm{km}_{2}$, the larger the value of $\chi$ is, the higher the rate of shale gas is (Fig. 6a); (2) $k_{\mathrm{m} 1}<=k m_{2}$, on the one hand, during the early flowing stage, the larger the value of $\chi$ is, the lower the rate of shale gas is, on the other hand, during the rest of flowing period, the larger the value of $\chi$ is, the higher the rate of shale gas is (Fig. 6b). From the analysis of micro-mechanisms, $k_{\mathrm{m} 1}<=$ $k_{\mathrm{m} 2}<k_{\mathrm{Fi}}$, as a result, the inter-porosity flowing capacity between matrix-2 and micro-fractures is stronger than that between matrix-1 and macro-fractures, therefore, the larger the pore volume ratio of matrix- 1 which depletes into macrofractures is, the less the total amount of shale gas from reservoir during the same productivity period is. In conclusion, as for the actual shale gas reservoir, we can acidize the matrix 


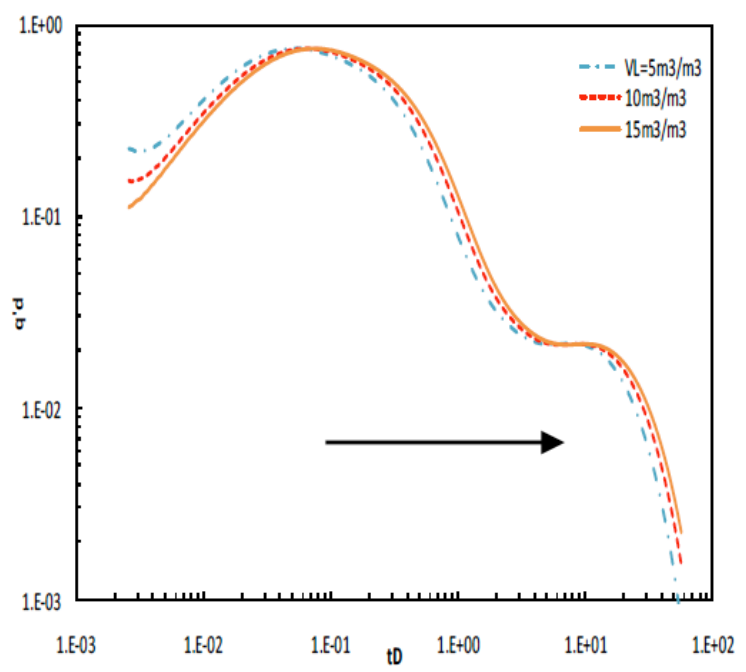

(a)

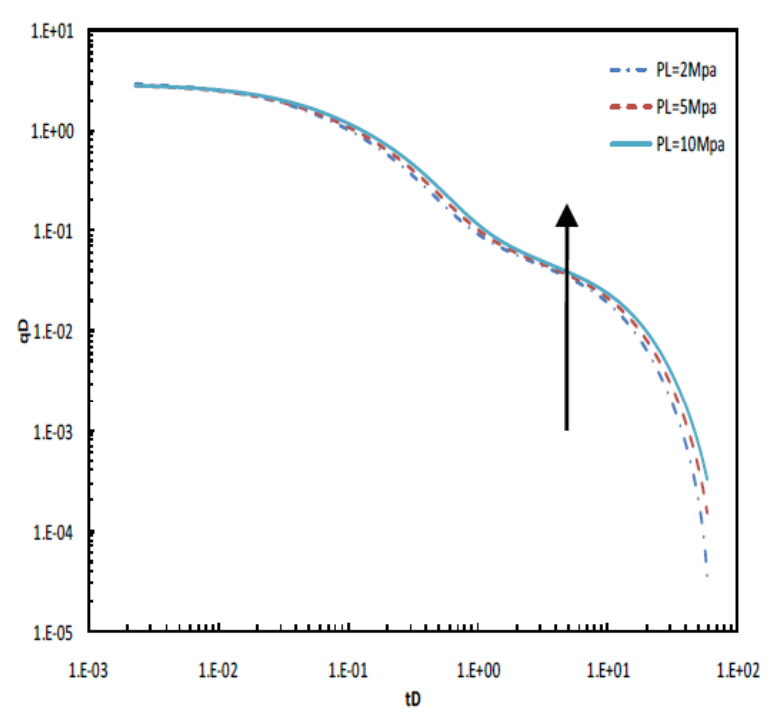

(c)

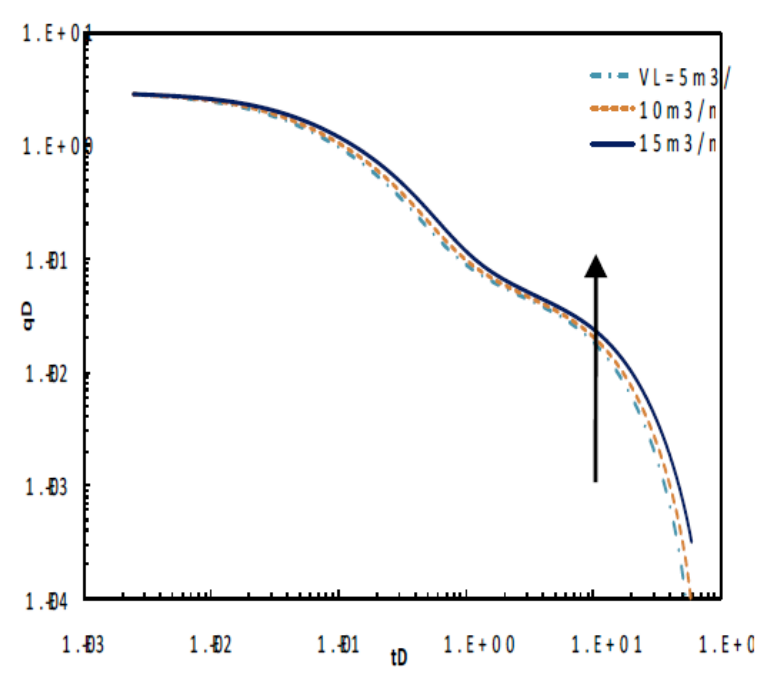

(b)

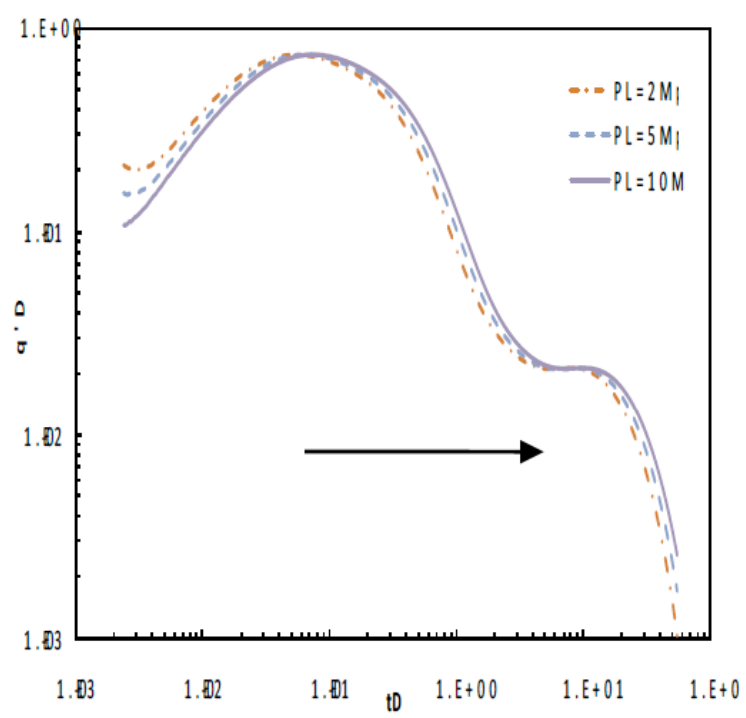

(d)

Fig. (5). Type curve under different value of Langmuir parameters. (a) $q_{D}$ vs $t_{D}$ under different Langmuir volume; (b) q'D vs $t_{D}$ under different Langmuir volume; (c) $\mathrm{q}_{\mathrm{D}} \mathrm{vs}_{\mathrm{D}} \mathrm{t}_{\mathrm{D}}$ under different Langmuir pressure; (d) $\mathrm{q}_{\mathrm{D}} \mathrm{vs}_{\mathrm{D}}$ under different Langmuir pressure

to increase its permeability so that the shale gas stored in matrix can directly flow into the macro-fractures, this treatment can significantly improve the horizontal well performance.

This paper assumes that USRV zone is dual-porosity slab model including matrix and fracture. The dimensionless rate type curves (Fig. 7) are plotted under different conditions in USRV zone, such as different matrix permeability, different fracture permeability and different width of reservoir. Observing from all of the following four figures, the rate rapidly declines without consideration of USRV zone. Generally speaking, the width of the whole reservoir is much larger than that of SRV zone; therefore, the flow occurs in the USRV zone can last longer than that in SRV zone (Fig. 7a). Fig. (7b) represents dynamic principle of rate under different permeability of matrix, the larger the value of ma- trix permeability is, the more the amount of gas deplete from matrix into fractures in USRV zone is, the later the boundary-dominated occurs. Fig. (7c) represents the dynamic principle of rate under different permeability of fracture in USRV zone, during the early productivity period, the larger the value of fracture permeability is, the more the gas rate is, however, the sooner the pressure wave reaches to the outer boundary, what's worse, the flowing regimes of matrix and micro-fractures in the these two zones are hardly observed (seen from the blue circle in Fig. 7c). Fig. (7d) reflects the impact of the width of reservoir, the wider the reservoir is, the more apparent the flowing regimes we can observe from the type curves. Through the above analysis, we can recognize that it is not reasonable to neglect the impact of USRV zone, especially for these kinds of reservoirs which have great formation properties. 


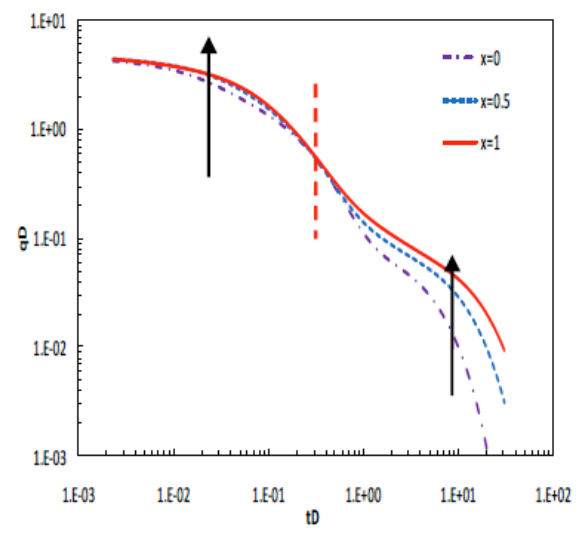

(a) $k \mathrm{~m}_{1}>k \mathrm{~m}_{2}$

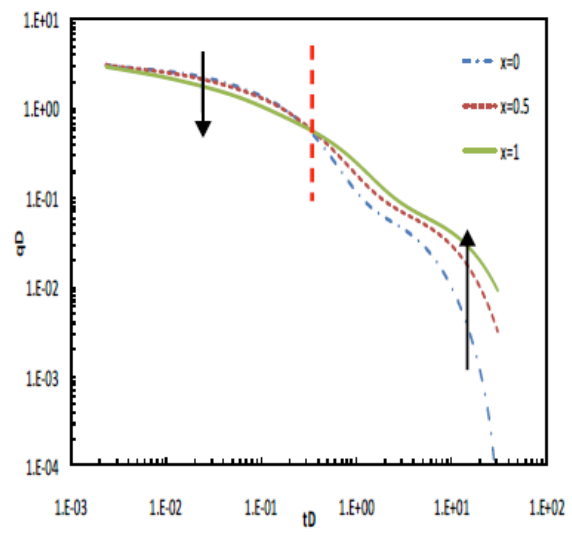

(b) $k \mathrm{~m}_{1}>k \mathrm{~m}_{2}$

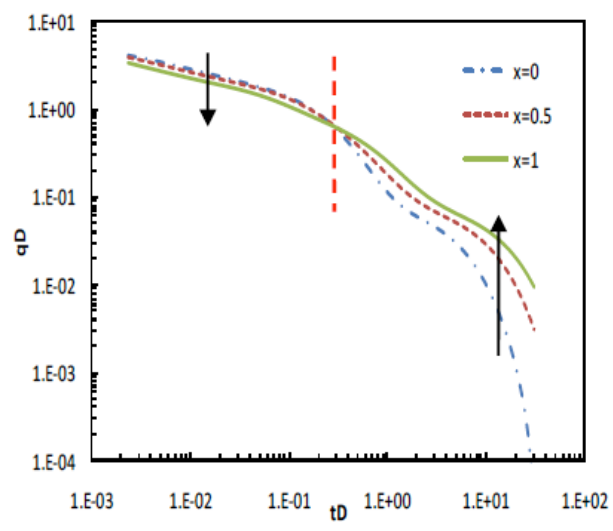

(c) $k \mathrm{~m}_{1}>k \mathrm{~m}_{2}$

Fig. (6). Type curve for the analysis of dual inter-porosity flowing.

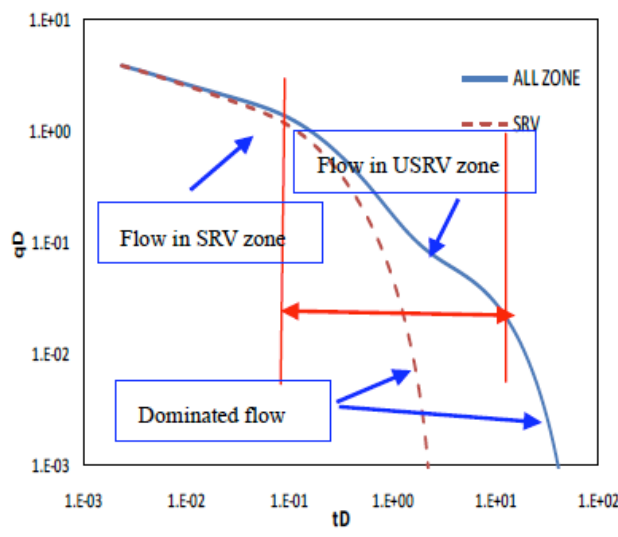

(a)

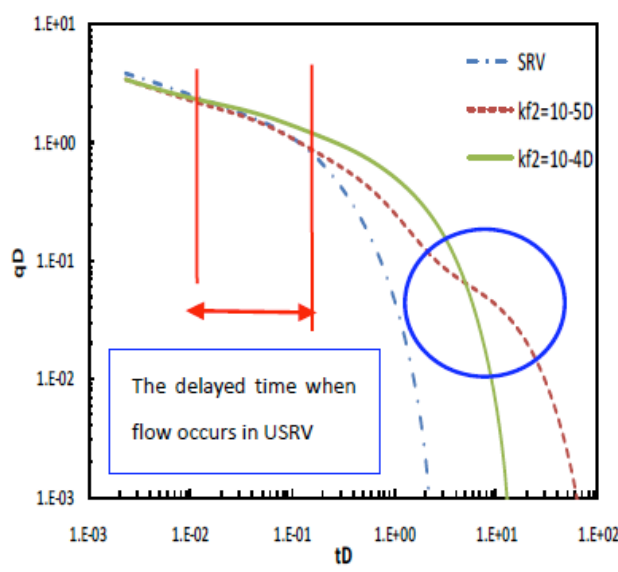

(c)

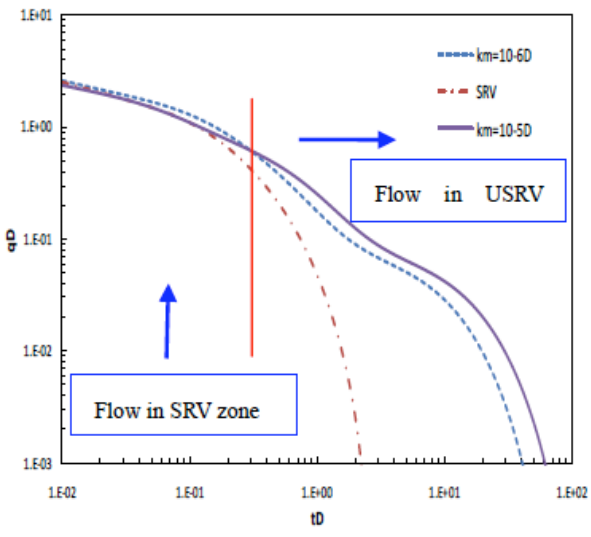

(b)

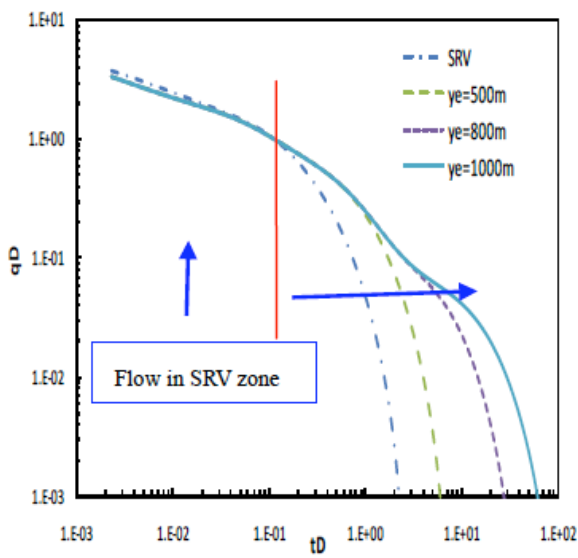

(d)

Fig. (7). Impact of USRV zone : (a) SRV zone and all zone; (b) different matrix permeability in USRV; (c) different fracture permeability in USRV; (d) different width of reservoir.

\section{CONCLUSION}

New analytical solution for shale gas reservoir with multi-stage fracturing horizontal well has been developed which considers the USRV zone as a dual porosity system and the SRV zone as a triple-porosity system. The solution is more general for type curve analysis both in homogeneous and naturally fractured reservoirs. Numerical simulation model was used to validate the analytical solutions and obtains an excellent agreement.

Analyzing the type curves, shale gas flow is divided into eight regimes: bilinear flow of macro-fractures and microfractures in SRV zone; pseudo-elliptic flow; linear flow of 
micro-fracture in SRV zone; dual-pseudo steady interporosity flow; transition flow between SRV and USRV zone; linear flow of fracture in outer zone; pseudo -steady state inter-porosity flow; outer boundary-dominated flow.

The impact of adsorption, stress sensitivity in shale gas reservoirs must not be ignored; otherwise, the performance of horizontal well cannot be predicted precisely. It is also concluded that the dual-porosity behavior of USRV zone has a positive effect on production, the larger the value of the permeability of matrix in USRV zone is, the more apparent the positive effect is. The stress sensitivity has negative influence on production during the whole productivity period.

This paper initially introduces the triple-porosity dualdepletion model in SRV zone for shale gas. It is concluded that the USRV zone has positive influence on production. Reservoirs could be acidized so as to increase the permeabil-

\section{NOMENCLATURE}

Cg gas compressibility, $\mathrm{MPa}^{-1}$

$C_{\mathrm{tF}}, C_{\mathrm{tf} 1}$ total compressibility of macro-fractures and microfractures in SRV zone, $\mathrm{MPa}^{-1}$

$C_{\mathrm{tm} 1}, C_{\mathrm{tm} 2}$ total compressibility of matrix in SRV zone $\mathrm{MPa}^{-1}$

$C_{\mathrm{tf} 2}$ total compressibility of micro-fractures in USRV zone, $\mathrm{MPa}^{-1}$

$C_{\mathrm{tm} 3}$ total compressibility of matrix in USRV zone, $\mathrm{MPa}^{-1}$

$h$ formation thickness, $\mathrm{m}$

$L$ horizontal well length, $m$

$y_{\mathrm{f}}$ macro-fracture length, $\mathrm{m}$

$y_{\mathrm{e}}$ reservoir width, $\mathrm{m}$

$\Phi_{\mathrm{F}}, \Phi_{\mathrm{fl}}$ porosity of macro-fractures and micro-fractures in

SRV zone

$\Phi_{\mathrm{m} 1}, \Phi_{\mathrm{m} 2}$ porosity of matrix in SRV zone

$\Phi_{\mathrm{f} 2}$ porosity of micro-fractures in USRV zone

$\Phi_{\mathrm{m} 3}$ porosity of matrix in USRV Zone

$k_{\mathrm{F}}, k_{\mathrm{f} 1}$ permeability of macro-fractures and micro-fractures in SRV zone, D

$k_{\mathrm{m} 1}, k_{\mathrm{m} 2}$ permeability of matrix in SRV zone, D

$k_{\mathrm{f} 2}$ permeability of micro-fractures in USRV zone, D

$k_{\mathrm{m} 3}$ permeability of matrix in USRV zone, D

$P_{\text {sc }}$ pressure at standard condition, $\mathrm{MPa}$

$q_{\mathrm{sc}}$ well production rate, $\mathrm{m}^{3} / \mathrm{d}$ ity of matrix in SRV zone and optimize the performance of horizontal wells for shale gas reservoirs.

\section{CONFLICT OF INTEREST}

The authors confirm that this article content has no conflict of interest.

\section{ACKNOWLEDGEMENTS}

The authors acknowledge a fund from the National Natural Science Foundation (NNSF) of China (No. 51204193) and supports from the MOE Key Laboratory of Petroleum Engineering. And this paper is supported by the fund NNSF of China (No. 51204193).

Notes: the program can provide if it is necessary.

s Laplace variable

$t$ time, h

$t_{\mathrm{D}}$ dimensionless time

$T$ temperature, $\mathrm{K}$

$T_{\text {sc }}$ temperature at standard condition, $\mathrm{K}$

$\Psi_{\mathrm{j}}$ pseudo pressure, $\mathrm{Mpa}^{2} / \mathrm{cp}, \mathrm{j}=\mathrm{F}, \mathrm{f} 1, \mathrm{~m} 1, \mathrm{~m} 2, \mathrm{f} 2, \mathrm{~m} 3$

$\mu$ viscosity, $\mathrm{cp}$

$\lambda_{\mathrm{fl}-\mathrm{F}}$ inter-porosity coefficient from mi-f to ma-f in SRV

$\lambda_{\mathrm{m} 2 \text {-f1 }}$ inter-porosity coefficient from $\mathrm{m} 2$ to mi-f in SRV

$\omega_{\mathrm{j}}$ storability coefficient, $\mathrm{j}=\mathrm{F}, \mathrm{f1}, \mathrm{m} 1, \mathrm{~m} 2, \mathrm{f} 2, \mathrm{~m} 3, \mathrm{~d}$

$x, y$ coordination, $\mathrm{m}$

$x_{\mathrm{D}}, y_{\mathrm{D}}$ dimensionless space

$q_{\mathrm{D}}$ dimensionless rate

$\chi$ pore volume ratio

$\mathrm{x}_{\mathrm{D}}, \mathrm{y}_{\mathrm{D}}$ dimensionless space

$A_{\mathrm{cw}}$ the area of wellbore, $2 \mathrm{Lh}, \mathrm{m}^{2}$

$\lambda_{\mathrm{m} 1-\mathrm{F}}$ inter-porosity coefficient from $\mathrm{m} 1$ to ma-f in SRV

subscript

D dimensionless

superscript

Laplace transform

, derivative

\section{APPENDIX A: DIMENSIONLESS DEFINITION}

Dimensionless pseudo pressure $\phi_{D}=\frac{\phi_{i}-\phi_{j}}{\phi_{i}-\phi_{w f}}, j=F, f_{1}, f_{2}, m_{1}, m_{2}, m_{3}$

Dimensionless time $t_{D}=\frac{3.6 k_{F i} t}{\mu \Lambda_{1} A_{c w}}, \Lambda_{1}=\left(\varphi c_{t}\right)_{F+f 1+m}+\frac{Z P_{s c} T V_{L} P_{L}}{T_{s c} \bar{P}\left(P_{L}+\bar{P}\right)^{2}}, m=m_{1}+m_{2}$

Dimensionless stress sensitivity factor $\beta_{D}=\beta\left(\phi_{i}-\phi_{w f}\right)$

Dimensionless space $y_{D}=\frac{y}{A_{c w}}, A_{c w}=2 x_{e} h, x_{D}=\frac{x}{L_{F} / 2}$ 
Dimensionless inter-porosity parameter

$$
\lambda_{f 1-F}=\frac{12}{L_{F}^{2}} \frac{k_{f 1}}{k_{F i}} A_{c w}, \lambda_{m 1-F}=\frac{12}{L_{F}^{2}} \frac{k_{m 1}}{k_{F i}} A_{c w},
$$

$$
\lambda_{m 2-f 1}=\frac{12}{L_{f}^{2}} \frac{k_{m 2}}{k_{F i}} A_{c w}, \lambda_{m 3-f 2}=\frac{12}{L_{F}^{2}} \frac{k_{m 3}}{k_{F i}} A_{c w}
$$

Dimensionless storativity ratio

SRV zone $\omega_{j}=\frac{\left(\varphi c_{t}\right)_{j}}{\Lambda_{1}},\left(j=F, f_{1}, m_{1}, m_{2}\right)$

Outer zone $\omega_{j}=\frac{\left(\varphi c_{t}\right)_{j}}{\Lambda_{2}},\left(j=f_{2}, m_{3}\right), \Lambda_{2}=\left(\varphi c_{t}\right)_{f_{2}+m_{3}}+\frac{Z P_{s c} T V_{L} P_{L}}{T_{s c} \bar{P}\left(P_{L}+\bar{P}\right)^{2}}$

Dimensionless conductivity ratio $\eta=\frac{k_{F i} / A_{c w}}{k_{f 1} /\left(L_{F} / 2\right)^{2}}$

Dimensionless production $q_{D}=\frac{8.589 \times 10^{-6} q_{s c} T}{\left(\phi_{i}-\phi_{w f}\right) k_{F i} \sqrt{A_{c w}}}$

Fracture permeability ratio $\tau=\frac{k_{F i}}{k_{f 2}}$

Transmissivity ratio $\sigma=\frac{\left(\varphi c_{t}\right)_{f_{2}+m_{3}}+\frac{Z P_{s c} T V_{L} P_{L}}{T_{s c} \bar{P}\left(P_{L}+\bar{P}\right)^{2}}}{\left(\varphi c_{t}\right)_{F+f_{1}+m}+\frac{Z P_{s c} T V_{L} P_{L}}{T_{s c} \bar{P}\left(P_{L}+\bar{P}\right)^{2}}}$

\section{APPENDIX B: SOLUTION OF EQUATIONS}

Applying the Laplace Transform, the governing equations and boundary conditions are changed into: SRV Zone:

macro-fracture: $\frac{d^{2} \bar{\zeta}}{d y_{D}^{2}}-\left.\frac{\lambda_{f 1-F}}{3} \frac{d \overline{\phi_{D f 1}}}{d x_{D}}\right|_{x_{D}=1}+2 \lambda_{m 1-F}\left(\overline{\phi_{D m 1}}-\overline{\phi_{D F}}\right)=\omega_{F} s \bar{\zeta}$

micro-fracture: $\frac{d^{2} \overline{\phi_{D f 1}}}{d x_{D}^{2}}+2 \lambda_{m 2-f 1} \eta\left(\overline{\phi_{D m 2}}-\overline{\phi_{D f 1}}\right)=\omega_{f 1} \eta s \overline{\phi_{D f 1}}$

matrix: $-2 \lambda_{m 1-F}\left(\overline{\phi_{D m 1}}-\overline{\phi_{D F}}\right)=\left(\omega_{m 1}+\omega_{d}\right) s \overline{\phi_{D m 1}}$

$-2 \lambda_{m 2-f 1}\left(\overline{\phi_{D m 2}}-\overline{\phi_{D f 1}}\right)=\left(\omega_{m 2}+\omega_{d}\right) s \overline{\phi_{D m 2}}$

USRV Zone:

fracture: $\frac{d^{2} \overline{\phi_{D f 2}}}{d y_{D}^{2}}+2 \lambda_{m 3-f 2}\left(\overline{\phi_{D m 3}}-\overline{\phi_{D f 2}}\right) \tau=\omega_{f 2} \sigma \tau s \overline{\phi_{D f 2}}$

matrix: $-2 \lambda_{m 3-f 2}\left(\overline{\phi_{D m 3}}-\overline{\phi_{D f 2}}\right)=\left(\omega_{m 3} \sigma+\omega_{d}\right) s \overline{\phi_{D m 3}}$

inner boundary condition:

$\left.\bar{\zeta}\right|_{y_{D}=0}=\frac{1-e^{-\beta_{D}}}{s \beta_{D}}$ 
interface condition:

$\bar{\zeta}\left(y_{D f}, s\right)=\overline{\phi_{D f 2}}\left(y_{D f}, s\right)$

$\left.k_{f 2} \frac{d \overline{\phi_{D f 2}}}{d y_{D}}\right|_{y_{D}=y_{f} / \sqrt{A_{c w}}}=\left.k_{F i} \frac{d \bar{\zeta}}{d y_{D}}\right|_{y_{D}=y_{f} / \sqrt{A_{c w}}}$

Inner zone fracture:

$$
\left.\frac{d \overline{\phi_{D f 1}}}{d x_{D}}\right|_{x_{D}=0}=0, \overline{\phi_{D f 1}}\left(x_{D}=1, s\right)=\bar{\zeta}
$$

Outer boundary condition:

$$
\left.\frac{d \overline{\phi_{D f 2}}}{d y_{D}}\right|_{y_{D}=y_{e} / \sqrt{A_{c w}}}=0
$$

Solving the SRV zone matrix equation:

$$
\begin{aligned}
& \overline{\phi_{D m 1}}=\frac{2 \lambda_{m 1-F}}{2 \lambda_{m 1-F}+\left(\omega_{m 1}+\omega_{d}\right) s} \overline{\phi_{D F}} \\
& \overline{\phi_{D m 2}}=\frac{2 \lambda_{m 2-f 1}}{2 \lambda_{m 2-f 1}+\left(\omega_{m 2}+\omega_{d}\right) s} \overline{\phi_{D f 1}}
\end{aligned}
$$

Substitute equation (B13) into (B2):

$$
\frac{d^{2} \overline{\phi_{D f 1}}}{d x_{D}^{2}}=f_{f 1}(s) \overline{\phi_{D f 1}}
$$

where:

$$
f_{f 1}(s)=\frac{2 \lambda_{m 2-f 1}\left(\omega_{m 2}+\omega_{d}\right) \eta s}{2 \lambda_{m 2-f 1}+\left(\omega_{m 2}+\omega_{d}\right) s}+\omega_{f 1} \eta s
$$

applied to the boundary condition:

$\overline{\phi_{D f 1}}=\frac{\bar{\zeta}}{\cosh \left(\sqrt{f_{f 1}(s)}\right)} \cosh \left(\sqrt{f_{f 1}(s)} x_{D}\right)$

Substitute equation (B16), (B12) into (B1):

$$
\frac{d^{2} \bar{\zeta}}{d y_{D}^{2}}=f_{F}(s) \bar{\zeta}
$$

where:

$f_{F}(s)=\frac{\lambda_{f 1-F}}{3} \sqrt{f_{f 1}(s)} \tanh \left(\sqrt{f_{f 1}(s)}\right)+\frac{2 \lambda_{m 1-F}\left(\omega_{m 1}+\omega_{d}\right) s}{2 \lambda_{m 1-F}+\left(\omega_{m 1}+\omega_{d}\right) s}+\omega_{F} s$

Solving the fracture equation in SRV zone:

$$
\frac{d^{2} \overline{\phi_{D f 2}}}{d y_{D}^{2}}=f_{f 2}(s) \overline{\phi_{D f 2}}
$$

where:

$f_{f 2}(s)=\tau \frac{2 \lambda_{m 3-f 2}\left(\sigma \omega_{m 3}+\omega_{d}\right) s}{2 \lambda_{m 3-f 2}+\left(\sigma \omega_{m 3}+\omega_{d}\right) s}+\omega_{f 2} \sigma \tau s$ 
applied to the interface condition:

$\bar{\zeta}=A \cosh \left(\sqrt{f_{F}(s)} y_{D}\right)+B \sinh \left(\sqrt{f_{F}(s)} y_{D}\right)$

where:

$A=\frac{1-e^{-\beta_{D}}}{s \beta_{D}} B=\frac{b \sinh \left(\sqrt{f_{F}(s)} y_{f} / \sqrt{A_{c w}}\right)-a \cosh \left(\sqrt{f_{F}(s)} y_{f} / \sqrt{A_{c w}}\right)}{a \sinh \left(\sqrt{f_{F}(s)} y_{f} / \sqrt{A_{c w}}\right)-b \cosh \left(\sqrt{f_{F}(s)} y_{f} / \sqrt{A_{c w}}\right)} \frac{1-e^{-\beta_{D}}}{s \beta_{D}}$

Applied to the Darcy law:

$\overline{q_{D}}=-\frac{b \sinh \left(\sqrt{f_{F}(s)} y_{f} / \sqrt{A_{c w}}\right)-a \cosh \left(\sqrt{f_{F}(s)} y_{f} / \sqrt{A_{c w}}\right)}{a \sinh \left(\sqrt{f_{F}(s)} y_{f} / \sqrt{A_{c w}}\right)-b \cosh \left(\sqrt{f_{F}(s)} y_{f} / \sqrt{A_{c w}}\right)} \frac{1-e^{-\beta_{D}}}{s \beta_{D}} \sqrt{f_{F}(s)}$

where:

$$
\begin{aligned}
& a=\frac{k_{f 2} \sqrt{f_{f 2}(s)}}{k_{F} \sqrt{f_{F}(s)}}\left[\sinh \left(\sqrt{f_{f 2}(s)} y_{f} / \sqrt{A_{c w}}\right)-\cosh \left(\sqrt{f_{f 2}(s)} y_{f} / \sqrt{A_{c w}}\right) \tanh \left(\sqrt{f_{f 2}(s)} y_{e} / \sqrt{A_{c w}}\right)\right] \\
& b=\left[\cosh \left(\sqrt{f_{f 2}(s)} y_{f} / \sqrt{A_{c w}}\right)-\sinh \left(\sqrt{f_{f 2}(s)} y_{f} / \sqrt{A_{c w}}\right) \tanh \left(\sqrt{f_{f 2}(s)} y_{e} / \sqrt{A_{c w}}\right)\right]
\end{aligned}
$$

\section{REFERENCES}

[1] C.R. Clarkson, "Production data analysis of unconventional gas wells: review of the theory and best practices", International Journal of Coal Geology, vol. 109-110, p. 101, 2013.

[2] E. Ozkan, M. Brown, R. Raghavan, and H. Kazemi, "Comparison of fractured horizontal-well performance in conventional and unconventional reservoirs", SPE Paper 121290-MS Presented at SPE Western Regional Meeting. San Jose, California, USA 2013.

[3] E. Stalgorova, and L. Mattar, "Practical model to simulate production of horizontal wells with branch fractures", SPE Paper 162515 Presented at SPE Canadian Unconventional Resource Conference, Calgary, Albert, Canada 2012a.

[4] E. Stalgorova, and L. Mattar, "Analytical model for history matching and forecasting production in multi-fractured composite systems", SPE Paper 162516-MS Presented at SPE Canadian Unconventional Resource Conference, Calgary, Albert, Canada 2012b.

[5] G.I. Barenblatt, I.P. Zhelto, and I.N. Kochina, "Bacis concepts of the theory of seepage of homogeneous liquids in fissured rocks', Journal of Applied Mathematical Mechanics., vol. 24, no. 2, p. 852, 1960.

[6] J.E. Warren, and P.J. Root, "The behavior of naturally fractured reservoirs", SPE Journal, vol. 3, no. 3, pp. 245, 1963.

[7] H. Kazemi, "Pressure transient of naturally fractured reservoirs with uniform fracture distribution", SPE Journal, SPE-2156A-PA, pp. 451-462, 1969.

[8] O.A. de Swaan, "Analytical solution for determining naturally fractured reservoir properties by well testing', SPE Journal, SPE5346-PA, pp. 117-122, 1976.

[9] E. Ozkan, M. Brown, R. Raghavan, and H. Kazemi, "Comparison of fractured horizontal well performance in tight sand and shale gas reservoirs", SPE Reservoir Evaluation \& Engineering, vol. 14, no. 2, pp. 248, 2011.

[10] A. Al-Ghamdi, and I. Ershaghi, "Pressure transient analysis of dually fractured reservoirs", SPE Journal, vol. 1, pp. 23, 1996.

[11] J.C. Liu, G.S. Bodvarsson, and Y.S. Wu, "Analysis of flow behavior in fractured lithophysal reservoirs", Journal of Contaminant Hydrology, vol. 62-63, pp.189, April-May 2003.

[12] Y.S. Wu, H.H. Liu, and G.S. Bodvarsson, "A triple-continuum approach for modeling flow and transport process in fractured rock", Journal of Contaminant Hydrology, vol. 73, pp. 145-179, 2004.

[13] J. Dreier, "Pressure transient analysis of wells in reservoirs with a multiple fracture network", MSc Thesis, Colorado School of Mines, Golden, Colorado 2004.

[14] A.H. El- Banbi, "Analysis of tight gas wells", PhD Dissertation, Texas 1998.

[15] H.A. Al-Ahmadi, and R.A. Wattenbarger, "Triple-porosity models: one further step towards capturing fractured reservoir heterogeneity", paper 149054-MS presented at the SPE/DGS Saudi Arabia Section Technical Symposium and Exhibition, 15-18 May 2011, AL-Khobar, Saudi Aribia: SPE, 2011.

[16] B.X. Xu, M. Haghighi, and X.F. Li, "Dennis Cooke. Development of new type curves for production analysis in naturally fractured shale gas/tight gas reservoirs", Journal of Petroleum Science and Engineering, vol. 105, p. 107, 2013.

[17] Y.L. Zhao, L.H. Zhang, J.Z. Zhao, J.X. Luo, and B.M. Zhang, "Triple porosity modeling of transient well test and rate decline analysis for multi-fractured horizontal well in shale gas reservoir", Journal of Petroleum Science and Engineering, vol. 12, no. 4, pp. 125, 2012.

[18] V.F. Samaniego, and H. Cinco-Leg, "Production rate decline in pressure sensitive reservoirs", Journal of Canadian Petroleum Technology, vol. 19, no. 3, pp. 75, 1980.

[19] R. Raghavan and L.Y. Chin, P"roductivity changes in reservoir with stress-dependent permeability, SPE Reservoir Evaluation and Engineering, vol. 7, no. 4, pp. 308, 2004.

[20] O.A. Pedrosa, Jr, "Petrobras. Pressure transient response in tresssensitive Formation, SPE, vol. 12, no. 3, pp. 213, 1986.

[21] H.T. Wang, "Performance of multiple fractured horizontal wells in shale gas reservoirs with consideration of multiple mechanisms", Journal of Hydrology, vol. 24, pp. 236, 2014.

[22] E.D. Obinna, and D. Hassan, "A model for simultaneous matrix depletion into natural and hydraulic fracture networks", Journal of Natural Gas Science and Engineering, vol. 16, pp. 57, 2014.

[23] H. Stehfest, "Numerical inversion of Laplace transforms-algorithm, Communications ACM, vol. 13, no. 1. pp. 47, 1970.

[24] H.T. Wang, "Performance of multiple fractured horizontal wells in shale gas reservoirs with consideration of multiple mechanisms", Journal of Hydrology, vol. 510, pp. 299-312, 2013.

Received: November 05, 2014

Revised: January 10, 2015

Accepted: January 21,2015

(C) Deng et al.; Licensee Bentham Open.

This is an open access article licensed under the terms of the Creative Commons Attribution Non-Commercial License (http://creativecommons.org/licenses/by-nc/3.0/) which permits unrestricted, non-commercial use, distribution and reproduction in any medium, provided the work is properly cited. 